\title{
MACROSOMÍA FETAL EN EL PERÚ PREVALENCIA, FACTORES DE RIESGO Y RESULTADOS PERINATALES
}

\author{
Responsable : Dr. Manuel Ticona Rendón \\ Miembro : : Mgr. Diana Huanco Apaza
}

\section{RESUMEN}

La macrosomia fetal ha sido definida como el feto grande para la edad de gestación (mayor del percentil 90) según incrementado riesgo perinatal que presenta.

Objetivo: Cuantificar la prevalencia de macrosomía fetal en hospitales del Ministerio de Salud del Perú e identificar sus factores de riesgo y resultados perinatales.

Material y Métodos: Se estudiarón 10966 recién nacidos macrosómicos en comparación con 75701 recién nacidos adecuados para la edad gestacional (AEG), clasificados según la curva de crecimiento intrauterino peruana, en 29 hospitales del Ministerio de Salud del Perú en el año 2005. Se estudiaron sus factores de riesgo y resultados perinatales utilizando Odds Ratio con intervalo de confianza. El procesamiento y análisis de información se realizaron con el Sistema Informático Perinatal.

Resultados: La prevalencia nacional de macrosomía fetal fue $11,37 \%$, las mismas que van desde 2,76\% en el Hospital Regional de Cajamarca hasta 20,91\% en el Centro de Salud Kennedy de Ilo. Los factores de riesgo significativos fueron: caracteristicas maternas: historia de macrosomía fetal $(O R=3,2)$, antecedente de diabetes $(O R=2,6)$, edad $>35$ años $(O R=1,4)$, talla $>1,65 \mathrm{~m}(\mathrm{OR}=2,75)$, peso $>65 \mathrm{~kg}(\mathrm{OR}=2,16)$, multiparidad $(O R=1,4)$ y las complicaciones maternas: polihidramnios $(O R=2,7)$, diabetes $(O R=1,7)$, hipertensión previa $(O R=1,4)$ y trabajo de parto obstruido $(O R=1,86)$. Los resultados perinatales fueron: mayor morbilidad $(O R=1,5)$, distocia de hombros $(O R=8,29)$, hipoglicemia $(O R=2,33)$, traumatismo del esqueleto $(O R=1,9)$, alteraciones hidro-electroliticas $(O R=1,9)$, asfixia al nacer $(O R=1,6)$ y traquipnea transitoria $(O R=1,5)$. No presentó mayor riesgo de mortalidad que los RNAEG.

Conclusiones: Los recién nacidos macrosómicos en el Perú tienen mayor riesgo de morbilidad, pero no presentan mayor mortalidad que los recién nacidos adecuados para la edad gestacional.

PALABRAS CLAVE: Macrosomia fetal; recién nacido grande para la edad gestacional.

\section{ABSTRACT}

The fetal macrosomia has been defined as the big fetus for the gestation (bigger than the percentile 90) age based on the perinatal increased risk that enters.

Objective: To quantify the prevalence of fetal macrosomia in hospitals of the Ministry of Health of Peru and to identify their factors of risk and perinatal results.

Material and Methods: 10,966 newborn macrosómicos was studied in comparison with 75,701 newly born adjusted for the gestational age (AEG), classified according to the growth curve intra-uterine Peruvian, in 29 hospitals of the Ministry of Health of Peru in the year 2005. It was studied their factors of risk and perinatal results using Odds Ratio with confidence interval. The indictment and analysis of information were carried out with the Data Perinatal System.

Results: The national prevalence of fetal macrosomia was $11,37 \%$, the same ones that go from $2,76 \%$ in the Regional Hospital of Cajamarca to 20,91\% in the Health centre Kennedy of Ilo. The significant factors of risk were: maternal features: history of fetal macrosomia $(O R=3,2)$, diabetes antecedent $(O R=2,6)$, age $>35$ years $(O R=1,4)$, it carves $>1,65 \mathrm{~m} .(O R=2,75)$, I weigh > $65 \mathrm{Kg} .(O R=2,16)$, multiparidad $(O R=1,4)$, and the maternal complications: polyhydramnioses $(O R=2,7)$, diabetes $(O R=1,7)$, previous $(O R=1,4)$ hypertension, and I work of clogged up childbirth $(O R=1,86)$. The perinatal results were: bigger morbilidad $(O R=1,5)$, dystocia of shoulders $(O R=8,29)$, hipoglicemia $(O R=2,33)$, traumatism of the skeleton $(O R=1,9)$, alterations hydro electrolytic $(O R=1,9)$, asphyxiates when being born $(O R=1,6)$ and transitory traquipnea $(O R=1,5)$. It didn't enter bigger risk of mortality that RNAEG.

Conclusions: The newborn macrosomics in Peru has bigger morbilidad risk but they don't enter bigger mortality that the newly born ones adjusted for the gestational age.

KEYWORD: Fetal macrosomia; Newborn big for the gestational age

\section{INTRODUCCIÓN}

El primer reporte de macrosomia fetal en la literatura fue hecho por el monje médico Francois Rabelais en el siglo XVI, quien relató la historia del bebé gigante Gargantúa. Muchos años después, la esposa de Gargantúa murió al parir a Pantagruel "porque era tan asombrosamente grande y pesado que no podia venir al mundo sin sofocar a su madre" (1).

Macrosomia o Macrosomatia (macro: 'grande'; soma: 'cuerpo'), etimológicamente significa tamaño grande del cuerpo. Tradicionalmente, la macrosomia fetal ha sido definida por un peso arbitrario al nacer, tal como 4000 ó 4500 gramos. El parto de estos fetos grandes ocasiona traumatismo tanto en la madre como en el feto. Históricamente, la macrosomia fetal ha estado asociada a una alta tasa de morbilidad y mortalidad materna y perinatal, dos veces mayor que la de la población general (2).

Los análisis de estadistica vitales han demostrado un incremento del peso al nacer a través del tiempo, siendo este incremento mayor en los países industrializados (3).

El peso al nacer de 4000 g. o más que representa aproximadamente al $5 \%$ de todos los nacimientos ha sido considerado en la mayoria de los estudios como sinónimo de 
macrosomía fetal. Por otro lado, el feto grande para la edad de gestación (GEG) que representa al $10 \%$ de la población general de recién nacidos, ha recibido hasta el momento poca atención por parte de los investigadores (4); es decir, un $5 \%$ de los fetos grandes no son clasificados en la actualidad como macrosómicos y no se benefician del enfoque de riesgo perinatal. Por lo tanto, la definición más correcta de macrosomia es la de considerar la edad gestacional y el percentil 90, los cuales tienen significativo mayor riesgo perinatal que los fetos de tamaño normal.

Desde el momento de la concepción cada fase del desarrollo humano está determinada por la interacción de los genes heredados y diversos factores ambientales. El peso de nacimiento en relación con la herencia se estima que, en alrededor del $70 \%$, está dado por factores de la madre. El peso de nacimiento es una caracteristica que ha preocupado permanentemente al equipo de salud de la atención primaria. Este, al sobrepasar los limites de normalidad, por déficit o por exceso, se convierte en un factor de riesgo para el niño, la madre 0 ambos. Por muchos años esta preocupación se concentró en el niño de bajo peso. Pero últimamente nos llama la atención que en una población de nivel socioeconómico medio bajo y bajo, como es la de nuestro Perú, hubiera una proporción de niños el doble de sobrepeso que de bajo peso (5).

Este hecho nos motivo para realizar un estudio con la finalidad de identificar la prevalencia, factores de riesgo de macrosomía fetal, conocer el resultado perinatal de estos neonatos comparado con la de los fetos de tamaño normal en 29 Hospitales del Ministerio de Salud del Perú

\section{MATERIALY MÉTODOS}

Durante el año 2005 ocurrieron 96444 partos atendidos en 29 hospitales del Ministerio de Salud del Perú, correspondiente a las tres regiones naturales del país. De ellos se estudiaron 10966 recién nacidos macrosómicos 0 grandes para la edad gestacional (GEG) en comparación con 75701 recién nacidos adecuados para la edad gestacional (AEG).

El tamaño por edad de gestación se determinó por la tabla de crecimiento intrauterina peruana recientemente propuesta. Se consideró feto adecuado para la edad de gestación (AEG) al recién nacido con peso entre el percentil 10 y 90 . Se consideró macrosomia fetal o feto grande para la edad de gestación (GEG) a todo feto con peso al nacer mayor o igual al percentil 90 .

Se determinó la prevalencia nacional de macrosomía fetal, así como por región natural y por hospital. Para la identificación de factores de riesgo, se realizó análisis bivariado, comparando los RN GEG y AEG empleando el Odds Ratio con intervalo de confianza, considerando significativo cuando este intervalo era mayor de 1. Para el procesamiento y análisis de datos se empleo el Sistema Informático Perinatal (SIP2000).

\section{RESULTADOS}

Durante el año 2005 nacieron 96444 recién nacidos vivos en 29 hospitales del Ministerio de Salud y de ellos 10 966 fueron macrosómicos, evaluados con la curva de crecimiento peruana, siendo la prevalencia nacional $11,37 \%$, las mismas que van desde 2,76\% en el Hospital Regional de Cajamarca hasta 20,91\% en el Centro de Salud Kennedy de llo.

Analizando la prevalencia por hospitales, podemos observar que en la región de la costa fue de 14,34\%; el Centro de Salud Kennedy de llo presentó la más alta $(20,91 \%)$ y el Hospital Regional de Ica la más baja $(11,29 \%)$; en la sierra la prevalencia fue de $7,12 \%$, siendo de $14,95 \%$ y $2,76 \%$ en los Hospitales de Moquegua y Regional de Cajamarca respectivamente; $y$ en la región de la selva fue de $9,81 \%$, el hospital Santa Rosa de Puerto Maldonado presentó la prevalencia más alta de $12,16 \%$ y el Hospital Regional de Loreto de 7,8\%. Tabla y Gráfico 1.

En relación con los factores de riesgo significativos para macrosomía fetal en el Perú, se encontró: antecedente de macrosomía fetal, antecedente de diabetes, edad mayor a 35 años, talla mayor de 1,65 m., peso pregestacional mayor de $65 \mathrm{~kg}$, multiparidad, polihidramnios, diabetes, hipertensión previa, trabajo de parto obstruido. Tabla 2.

Tabla N 01: Prevalencia de la macrosomia fetal según curvas de crecimiento peruanas, hospitales del ministerio de salud del Perú 2005.

\begin{tabular}{|c|c|c|}
\hline & HOSPITAL & GEG $\%$ \\
\hline & 29 HOSPITALES MINSA PERU & 11,37 \\
\hline \multirow{10}{*}{ 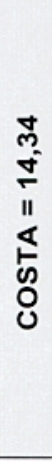 } & C.S. Kennedy de llo & 20,91 \\
\hline & H. Hipólito Unanue de Tacna & 20,83 \\
\hline & H. Regional Docente de Trujillo & 14,03 \\
\hline & H. Nacional Hipólito Unanue & 13,40 \\
\hline & Instituto Materno Perinatal & 13,36 \\
\hline & H. San Bartolome de Lima & 13,18 \\
\hline & H. María Auxiliadora de Lima & 12,78 \\
\hline & H. Belén de Trujillo & 12,30 \\
\hline & H. de Apoyo de Sullana & 11,43 \\
\hline & H. Regional de Ica & 11,29 \\
\hline \multirow{13}{*}{ 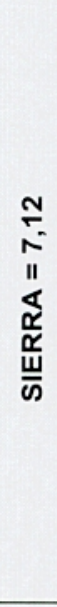 } & H. de Moquegua & 14,95 \\
\hline & H. Goyeneche de Arequipa & 11,89 \\
\hline & H. Hermilio Medrano de Huánuco & 10,77 \\
\hline & H. Subregional de Andahuaylas & 8,40 \\
\hline & H. M. Núñez Butrón de Puno & 7,29 \\
\hline & H. Regional del Cusco & 6,46 \\
\hline & H. D. Alcides Carreon de Huancayo & 6,42 \\
\hline & H. Regional de Ayacucho & 6,04 \\
\hline & H. Victor Ramos de Huaraz & 5,28 \\
\hline & H. Antonio Lorena del Cusco & 5,23 \\
\hline & H. de Apoyo de Huancavelica & 3,72 \\
\hline & H. El Carmen de Huancayo & 3,32 \\
\hline & H. Regional de Cajamarca & 2,76 \\
\hline \multirow{6}{*}{ 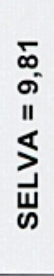 } & H. Santa Rosa de P. Maldonado & 12,16 \\
\hline & H. de Apoyo de lquitos & 11,12 \\
\hline & C. Materno Perinatal de Tarapoto & 9,72 \\
\hline & H. Regional de Pucallpa & 9,66 \\
\hline & H. de Apoyo Yarinacocha & 8,39 \\
\hline & H. Regional de Loreto & 7,80 \\
\hline
\end{tabular}


Figura $\mathrm{N}^{\circ} 01$

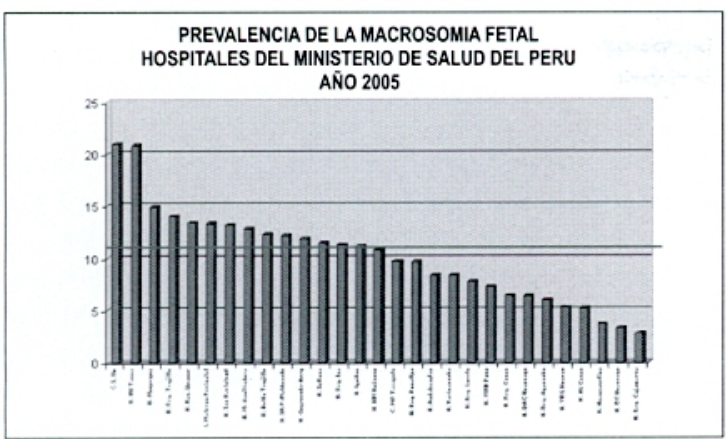

Tabla N 02 : Factores de Riesgo de La Macrosomia Fetal Hospitales del Ministerio de Salud del Perú -2005

\begin{tabular}{|c|c|c|c|c|c|c|}
\hline \multirow{2}{*}{$\begin{array}{c}\text { Factor De } \\
\text { Riesgo }\end{array}$} & \multicolumn{2}{|c|}{ GEG } & \multicolumn{2}{|c|}{ AEG } & \multicolumn{2}{|c|}{ RIESGO } \\
\hline & $F$ & $\%$ & $\mathrm{~N}$ & $\mathrm{~s}$ & $O R$ & IC \\
\hline \multicolumn{7}{|l|}{ Caracteristicas Matermas } \\
\hline Historia Feto Macrosímico & 911 & 21,00 & 1864 & 7,54 & 3.26 & $299-3.55$ \\
\hline Talala 1.65 M A M/ss & 717 & 6,54 & 3270 & 3,78 & 2.75 & $1.96-3.82$ \\
\hline Artec. Pers. Diabeles & 15 & 0,14 & 39 & 0.05 & 2.66 & $1,46-4.82$ \\
\hline Pesso 65 Kg. AMbas & 2554 & 26.97 & 9257 & 14,62 & 2.16 & $2,05 \cdot 2.27$ \\
\hline Diabeles & 25 & 0,23 & 100 & 0,13 & 1,73 & $1,11-260$ \\
\hline Mitjpara (4AMas) & 1406 & 1288 & 6810 & 9,00 & 1,49 & $1,40-1,58$ \\
\hline$E d x d>35$ A $A^{5}=5$ & 1793 & 18.93 & 8430 & 13,91 & 1,45 & $1,37-1.53$ \\
\hline \multicolumn{7}{|l|}{ Complicaciones Mrtemas } \\
\hline Porhidramnics & 50 & 0,46 & 126 & 0,17 & 2,75 & $1.96-3.82$ \\
\hline Tricaio D Paro Obstruido & 274 & 2.50 & 1027 & 1,36 & 1.86 & $1,63-2,13$ \\
\hline Cestrea & 4245 & 38,71 & 21174 & 27,97 & 1.63 & $1,50-1,70$ \\
\hline Hipertension Atrerial & 42 & 0,38 & 195 & 0.26 & 1,49 & $1.07-2.08$ \\
\hline
\end{tabular}

Tabla N 03 : Resultados Perinatales de la Macrosomía Fetal Hospitales del Ministerio de Salud del Perú - 2005

\begin{tabular}{|l|c|c|c|c|c|c|}
\hline \multirow{2}{*}{ RIESGO } & \multicolumn{2}{|c|}{ GEG } & \multicolumn{2}{c|}{ AEG } & \multicolumn{2}{c|}{ RIESGO } \\
\cline { 2 - 7 } & $N^{2}$ & $\%$ & $N^{\circ}$ & $\%$ & OR & IC \\
\hline Morblidad Neonatal & 1140 & 10,40 & 5438 & 7,18 & 1,50 & $1,40-1,60$ \\
\hline Mortalidad Neonatal & 74 & 0,87 & 497 & 0,81 & 1,06 & $0,83-1,36$ \\
\hline Distocia de hombros & 6 & 0,05 & 5 & 0,01 & 8,29 & $2,53-37,18$ \\
\hline Hipogicenia & 72 & 0,66 & 214 & 0,28 & 2,33 & $1,78-3,05$ \\
\hline Truumatismo esquéético & 25 & 0,23 & 87 & 0,11 & 1,99 & $1,27-3,10$ \\
\hline $\begin{array}{l}\text { Alterac. } \\
\text { hidroelectroliticas }\end{array}$ & 53 & 0,48 & 184 & 0,24 & 1,99 & $1,47-2,71$ \\
\hline Asfixia al nacer & 54 & 0,49 & 231 & 0,31 & 1,62 & $1,20-2,18$ \\
\hline Taquipnea transitoria & 45 & 0,41 & 200 & 0,26 & 1,56 & $1,12-2,15$ \\
\hline
\end{tabular}

Tabla No 04 : Riesgos Atribuibles Poblacionales de la Macrosomia Fetal en Hospitales del Ministerio de Salud del Perú.

\begin{tabular}{|l|c|}
\hline \multicolumn{1}{|c|}{ FACTORES DE RIESGO } & RAP \& \\
\hline Historia de feto macrosómico & 13 \\
\hline Peso pregestacional $65 \mathrm{~kg}$. a más & 7 \\
\hline Edad materna 35 años a más & 5 \\
\hline Multipara $(4$ a ) & 4 \\
\hline Talla materna 1,65 m. a más & 3 \\
\hline
\end{tabular}

\section{DISCUSIÓN}

La prevalencia de macrosomia fetal en 29 Hospitales del Ministerio de Salud del Perú fue de $11,37 \%$, oscilando entre $7,12 \%$ en la sierra, $9,81 \%$ en la selva y $14,34 \%$ en la costa, $y$ a nivel hospitalario entre $2,76 \%$ y $3,32 \%$ en los Hospitales de Cajamarca y el Carmen de Huancayo ubicados en la sierra y $20,91 \%$ y $20,83 \%$ en el Centro de Salud Kennedy de llo y Hospital de Tacna en la costa.

La prevalencia encontrada en nuestro estudio no hace más que reafirmar que la macrosomía fetal está asociada fundamentalmente a las condiciones socio económico de la población.

A nivel internacional, sólo encontramos estudios hospitalarios, como López (5) entre 1997 y 2000 en Santiago de Chile encuentra macrosomía entre 6,6\% y $13 \%$. Ballesté
(6) en el hospital Ginecoobstétrico docente de Guanabacoa en Cuba encontró el indice de macrosomía fetal de 3,7\%. Martinez en la Clínica las Condes de Santiago de Chile 7\%. Cutié (8) en el Hospital Militar Central "Dr. Luis Diaz Soto" en Cuba de 1996 a 2001, 4,7\%. Joyar (9) en el Hospital Materno Infantil $1^{\circ}$. De Mayo en el Instituto Salvadoreño de Seguro Social en el año 2004, 3,1\%. La Fontaine (10) en el Hospital Provincial Docente Obstétrico Ana Betancourt de Mora de Camaguey en Cuba de 1999 a 2004, 7,36\%. Giusti (11) en el Hospital Llano de la ciudad de Corrientes en Argentina, encontró en 1999 al 2001 una incidencia de 5,17\%. Salazar (12) en el Hospital Adolfo Prince Lara en Puerto Cabello en el Estado de Carabobo de Venezuela en 1993,3,76\%. Albornoz (13) en la maternidad del Hospital Clínico de la Universidad de Chile del 2001 al 2003, encontró una incidencia de 12,6\%.

A nivel nacional, Pacora (14) entre 1990 y 1993, en el Hospital San Bartolomé de Lima encontró macrosomia fetal de $16,4 \%$ utilizando las tablas de Brenner (15).

Las características maternas asociadas a macrosomía fetal en el Perú encontradas en este estudio fueron: historia de feto macrosómico, talla y peso alto, multiparidad y edad mayor e igual a 35 años, antecedentes personales de diabetes y diabetes en el embarazo. Entre las complicaciones maternas se encontraron hipertensión arterial, polihdramnios, trabajo de parto obstruido y cesárea.

Comparando los factores de riesgo de la macrosomía fetal a nivel nacional, Pacora (14) en el Hospital San Bartolomé, encuentra como factores predictivos de macrosomia fetal, en orden de especificidad: ganancia ponderal excesiva, feto de sexo varón, edad mayor de 30 años, obesidad, antecedente de feto grande, talla mayor de $1,60 \mathrm{~m}$, diabetes familiar y anemia.

A nivel internacional se encontraron los siguientes factores de riesgo: Salazar en Venezuela (12) encontró como factores más importantes: antecedentes maternos como recién nacido macrosómico, diabetes, hipertensión arterial, las patologias asociadas más frecuentes fueron: diabetes, ganancia de peso materno de más de $15 \mathrm{~kg}$., enfermedad hipertensiva, embarazo prolongado, las complicaciones que se presentaron en el parto fueron: desgarro de partes blandas, distocias del parto, trabajo de parto prolongado y retención de hombros.

Albornoz (13), en Chile, encontró que la incidencia global de traumatismo asociada al parto fue significativamente más alta en parto vaginal operatorio y vaginal normal en relación con cesáreas. El traumatismo obstétrico en RN macrosómicos estaba en relación directa con el peso del RN y su mortalidad intraparto, concluyendo que la macrosomía fetal es un importante predictor de parto cesárea y de una mayor morbilidad neonatal y mortalidad fetal intraparto. López (5), en Chile, encuentra que los niños macrosómicos en comparación con los nacidos de peso normal, difieren significativamente en mayor edad materna, mayor escolaridad, mayor peso al inicio y término de la gestación, antecedente de diabetes materna y mayor parto instrumental.

Ballesté (6), en Cuba, halló asociación con edad materna mayor de 30 años, edad gestacional mayor de 42, talla materna con $1,70 \mathrm{~m}$, antecedente de hijo macrosómico 
anterior, diabetes familiar y sexo masculino. Martinez (7), en Chile, no encontró asociación con trauma obstétrico ni aumento de cesárea por macrosomía fetal. Joyar (9), en Salvador, encuentra que la vía de evacuación de los productos macrosómicos no afecta el pronóstico obstétrico ni neonatal, es decir, que no existen diferencias entre las complicaciones maternas y neonatales entre el nacimiento por vía vaginal o cesárea, como distocia de hombros, atonía uterina, distress respiratorio y Apgar bajo al nacer. Cutié (8), en Cuba, encuentra que los principales antecedentes maternos asociados a macrosomia fetal fueron embarazo prolongado, diabetes, obesidad y multiparidad.

El 10,4\% de los RN macrosómicos presentó morbilidad neonatal en comparación con $7,18 \%$ de los RN de peso adecuado, teniendo 1,5 veces mayor riesgo de morbilidad, no hubo diferencias en la tasa de mortalidad neonatal. Entre las patologias asociadas a macrosomía encontramos: distocia de hombros, hipoglicemia, traumatismo del esqueleto, asfixia al nacer y taquipnea transitoria. No hemos encontrado asociación con elongación de plexo braquial, Apgar bajo a los 5 minutos, septicemia, traumatismo del cuero cabelludo, hipocalcemia, ictericia, policitemia ni sindrome aspirativo.

Entre los resultados perinatales asociados a macrosomia fetal a nivel nacional, Pacora (14), encontró en forma significativa mayor morbilidad perinatal, principalmente distocia de hombros, pero no hubo diferencia en la tasa de mortalidad perinatal.

A nivel internacional, Salazar (12), en Venezuela, encontró mayor morbilidad como: sufrimiento fetal agudo, hipoxia perinatal, hipoglicemia y parálisis braquial, encontró mayor mortalidad fetal pero no encontró mortalidad neonatal. López (5), en Chile, no encontró complicaciones como distocia de hombros, distress respiratorio, hipoglicemia. Ballesté (6,) en Cuba, no encontró mayor riesgo de cesáreas, ni de Apgar bajo. Cutié (8), en Cuba, no encuentra asociación a Apgar bajo ni hubo mortalidad fetal ni neonatal y las complicaciones que con mayor frecuencia se encontraron en el RN macrosómico fueron distocias de hombros y elongación de plexo braquial.

A pesar del adelanto tecnológico, el trauma obstétrico sigue siendo un problema en nuestros hospitales debido a la dificultad de identificar la macrosomia fetal antes del parto. Asi, los partos vaginales con distocia de hombros complican 8 veces más que los neonatos con peso adecuado. Esto ocasiona mayor riesgo de alteraciones metabólicas, principalmente hipoglicemia, asfixia neonatal, traumatismo esquelético y taquipnea transitoria, y la consecuente necesidad del ingreso de estos niños a la unidad de cuidados especiales.
El diagnóstico de macrosomía fetal depende en gran medida de la sospecha clínica. Asi, en este estudio hemos encontrado que todas las gestantes presentaron algún factor de riesgo identificable antes del parto. Sin embargo, reportes extranjeros señalan que sólo $40 \%$ de los macrosómicos pueden identificarse por factores de riesgo materno. El diagnóstico es incorrectamente hecho en $7 \%$ de bebés de tamaño normal. Por cada correcta identificación de feto macrosómico hubo nueve falsos positivos (14). Creemos que esto obedece a la falta de una buena evaluación prenatal donde no se identifican factores de riesgo en forma oportuna.

Toda esta información nos permite señalar que la macrosomia fetal es un predictor de riesgo en la salud futura de la madre y el niño, por lo que debemos de evitarla.

\section{BIBLIOGRAFÍA}

Langer 0. Fetal Macrosomia: Etiological Factors. In Abnormal Fetal Growth, pp. 99110. Edited by M.Y. Divon. Elsevier Science Publishing Co, Inc. New York, Amsterdam, London 1991.

Modanlou HD, Dorchester WIL, Thorosina A, Frreeman RK: Macrosomia maternal, fetal and neonatal implications. Obstet Gynecol 1980; 55: 420-424.

Hytten F and Leitch I. Birth weight. In the Physiology of Human Pregnancy pp. 240259. Blackwell Scientific Publication, Oxford London 1964.

Varner MW, Disproportionate Fetal growth. Chapter 15 in Current Obstetric \& Gynecologic Diagnosis \& Treatment, pp. 340351. Edited by M.L. Pernol. 7th Edition. Appleton \& Lange. USA 1991.

López I, Sepúlveda H, Jeria C, Letelier C. Niños macrosómicos y de peso normal en un consultorio de atención primaria. Comparación de características propias y maternas 1997 - 2000. Rev Chil Pediatr. 2003; 74 (3): 287 293.

Ballesté I, Alonso RM. Factores de riesgo del recién nacido macrosómico. Rev. Cubana de Pediatría. 2004; 76 (1).

Martínez J, Pardo J. Macrosomía fetal. Riesgo Perinatal. Revista Médica de Clínica Las Condes. Santiago de Chile. Abril 2003; 14 (2).

Cutié M, Figueroa M, Segura A, Lestayo C. Macrosomia fetal. Su comportamiento en el último quinquenio. Rev Cubana Obstet Ginecol. Enero a abril 2002; 28 (1).

Joyar A. Macrosomía fetal. Morbimortalidad obstétrica yneonatal. 